\title{
The AEROARMS Project: Aerial Robots with Advanced Manipulation Capabilities for Inspection and Maintenance
}

\author{
Anibal Ollero, Guillermo Heredia, Antonio Franchi, Gianluca Antonelli, Konstantin Kondak, Alberto Sanfeliu, \\ Antidio Viguria, J.Ramiro Martinez-de Dios, Francesco Pierri, Juan Cortés, Angel Santamaria-Navarro, \\ Miguel Angel Trujillo, Ribin Balachandran, Juan Andrade-Cetto and Angel Rodriguez
}

\begin{abstract}
This paper summarizes new aerial robotic manipulation technologies and methods, required for outdoor industrial inspection and maintenance, developed in the AEROARMS project. It presents aerial robotic manipulators with dual arms and multi-directional thrusters. It deals with the control systems, including the control of the interaction forces and the compliance, the teleoperation, which uses passivity to tackle the tradeoff between stability and performance, perception methods for localization, mapping and inspection, and planning methods, including a new control-aware approach for aerial manipulation. Finally, it describes a novel industrial platform with multidirectional thrusters and a new arm design to increase the robustness in industrial contact inspections. The lessons learned in the application to outdoor aerial manipulation for inspection and maintenance are pointed out.
\end{abstract}

Index Terms-aerial robotic manipulation, aerial robotics, inspection and maintenance.

\section{INTRODUCTION}

A ERIAL manipulation deals with aerial robots equipped with arms (see Fig. 1) or other end-effectors performing tasks, such as assembly or contact inspection, in locations that are inaccessible, very dangerous or costly to be accessed from ground. It involves very challenging problems, mainly due to aerodynamic influence and the fast dynamics when compared to other floating-base manipulation systems.

Most of the works in aerial robotic manipulation have been performed indoors, with very few exceptions such as grasping of objects from the air with a helicopter [1]. These indoor works included picking and basic manipulation with quadrotors and also cooperative assembly with several aerial robots [2]. In [3], a quadrotor with a small arm maintaining contact with a surface was reported. Indoor redundant manipulators

A. Ollero, G. Heredia, R. Martinez-de Dios and A. Rodriguez are with Universidad de Sevilla, Sevilla, Spain, \{aollero, guiller, jdedios, castano\}@us.es

A. Franchi and J. Cortés are with LAAS-CNRS, Université de Toulouse, CNRS, Toulouse, France, \{antonio.franchi, juan.cortes\}@laas.fr

F. Pierri is with the University of Basilicata, School of Engineering, via dell'Ateneo Lucano, 10, Potenza, Italy, francesco.pierri@unibas.it

G. Antonelli is with the University of Cassino and Southern Lazio, Via Di Biasio 43, 03043 Cassino, Italy, gianluca.antonelli@unicas.it

K. Kondak and R. Balachandran are with DLR, Germany, \{konstantin.kondak, Ribin.Balachandran\}@dlr.de

A. Sanfeliu, J. Andrade-Cetto and A. Santamaria-Navarro are with Institut de Robotica i Informatica Industrial, CSIC-UPC, Barcelona, Spain, \{asantamaria, cetto, sanfeliu\}@iri.upc.edu

A. Viguria and M.A. Trujillo are with CATEC, Spain, \{aviguria, matrujillo\}@catec.aero

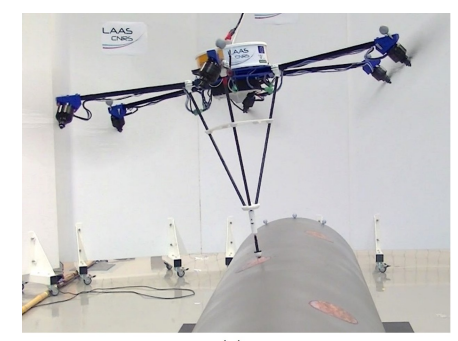

(a)

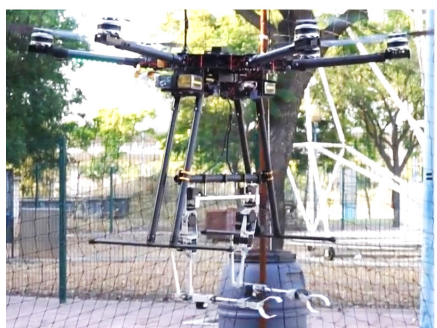

(c)

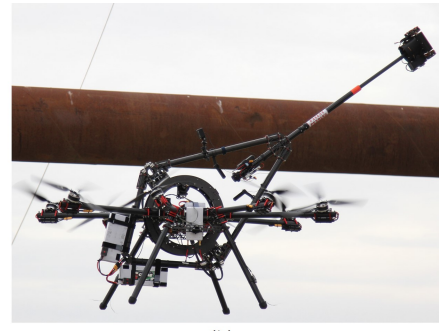

(b)

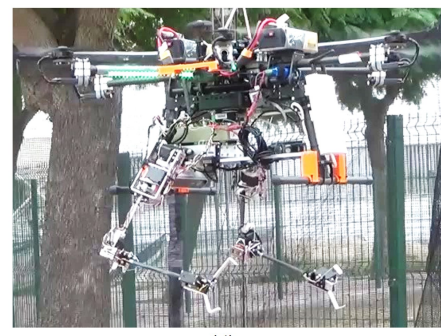

(d)
Fig. 1. AEROARMS platforms: (a) Multi-directional thrust hexarotor with a rigidly attached end-effector; (b) Multi-directional thrust octorotor with an inspection arm; (c) Aerial dual-arms manipulator with stiff joints; (d) Aerial dual-arms manipulator with compliant joints

are studied in [4]. In [5], an indoor flying robot capable of depositing polyurethane foam is presented. Later on, new indoor aerial manipulators were presented and demonstrated for applications such as drawer opening [6]. Compliance was studied in [7]. The transportation of bars with two quadrotors was presented in [8]. More recently new aerial grasping [9] and, in general, aerial systems interacting with the environment have been presented [10]. All these systems have been demonstrated indoor. In the ARCAS project (http://www.arcasproject.eu/), indoor and outdoor aerial manipulator prototypes with both helicopters (equipped with the KUKA-DLR LWR arm) and multi-rotor systems (equipped with light arms) were demonstrated, including fully autonomous visual servoing. In AEROWORKS (http://www.aeroworks2020.eu/) collaborative exploration, aerial manipulation, and also estimation, localization and control of UAVs are included. Aerial Manipulation has been also demonstrated indoors [11].

On the other hand, the AEROARMS project (https://aeroarms-project.eu/) is more focused on aerial manipulation for the outdoor application to Inspection and 


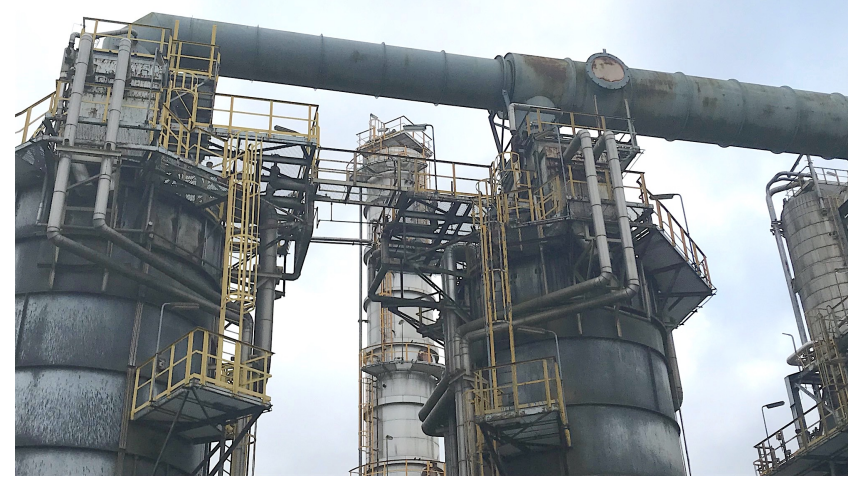

Fig. 2. Oil and Gas refinery in Germany.

Maintenance (I\&M) and it includes the development of new aerial robotic manipulators. Three use cases in industrial environments, particularly in Oil \& Gas plants (see Fig. 2) were selected: direct contact measurements while flying, robotic crawler deployment, and installation of sensors. Performing experiments in these plants require previous extensive outdoor testing with reliable aerial manipulators able to deal with the harder outdoor constraints in industrial settings including the agility to react to wind perturbations, compliance to afford unexpected impacts, variations in lighting conditions, and the need to compensate for inaccuracies in GNSS positioning.

Solving the above inspection use-cases in realistic settings is a hard problem. We believe the design and development of such manipulation systems requires the following developments:

- Aerial robotic manipulators able to apply forces for contact inspection in any direction, ability to compensate wind perturbations, as well as dual arm manipulators for the installation of sensors in complex settings;

- Control systems for the above aerial manipulators, integrating the aerial platform and the manipulator;

- Bilateral teleoperation systems with haptic interfaces and appropriated trade-off between stability and performance;

- Reliable outdoor autonomous perception system;

- Planning system considering the dynamic behavior in closed loop of the aerial platform and the arm.

The above components are summarized in the following sections. The paper also presents a new robot for industrial applications, the lessons learned and the conclusions.

\section{PLATFORMS}

Aerial manipulators are multi-body systems with coupled (aerial platform-manipulator) dynamic behavior applying forces to objects. AEROARMS includes the modeling and control of propulsion systems [12] and aerodynamic effects when flying very close to the environment [13], but this paper concentrates on the main design aspects of platforms.
Most aerial manipulators [3]-[6] use standard multi-rotor platforms with all propellers oriented in the same direction. They are thus underactuated with only four DoF, and they need to tilt to move or exert forces in the horizontal plane. Non-conventional multi-directional thrust platforms with tilted rotors [14] have been designed with the capability of directing the total thrust in more than one direction in body frame (see Fig. 1(a)), thus capable of full 6D wrench exertion without tilting. This configuration has been used in the Tilt-Hex hexarotor platform (Fig. 1(a)) and in the AEROX octorotor industrial platform (Fig. 1(b)). The characteristics of these platforms are shown in Table 1.

Aerial manipulators use mechatronic devices as bars, grippers or multi-link arms to reach the operation point, grasp and manipulate objects and exert forces on the environment. The design of these devices is important since their movement induce the displacement of the Center off Mass (CoM) and variation of moments of inertia, generating disturbance torques and affecting the dynamics of the aerial vehicle [4]. To overcome these effects, the AEROX new arm configuration has been designed. The arms weight is compensated with the batteries, thus the movement of the arm does not modify the position of the CoM of the aerial manipulator. Additionally, any contact forces from the interaction with the environment are transmitted directly to the CoM without generating perturbation torques.

More advanced manipulation than simple grasping or exerting forces over a surface requires the use of aerial manipulators with dual arms. Very few dual arm aerial manipulators have been developed, as for example [15], which use two 3-DoF arms for valve turning. In AEROARMS, two new anthropomorphic dual-arms aerial manipulators with 4 and 5 joints (see Table 1) have been developed, since this configuration maximizes dual arm manipulability. The first one is an octorotor with arms of $0.9 \mathrm{Kg}$, and the maximum load that can be lifted by the two arms with stiff joints is $0.7 \mathrm{~kg}$ (Fig. 1(c)). Taking into account that collisions and impacts can severely affect stability of the aerial manipulators, and compliance has been found to be very useful [7], a dual arm aerial manipulator with compliant joints [16] has been developed and tested outdoor (Fig. 1(d)). It is a hexarotor and the arms have joints with elastic elements that can absorb contact and collision forces generated by physical interaction, providing a compliant behavior that increases robustness and stability and also enables measuring interaction forces for force control. The total weight for both arms is $1.3 \mathrm{~kg}$ and the maximum load that each arm can lift is $0.2 \mathrm{~kg}$.

\section{CONTROL Systems}

One of the key advances of AEROARMS has been to deeply investigate the potentiality and limitations of control architectures for aerial manipulation. More in detail, as far as the interaction control is concerned, the solution based on a multi-directional thruster platform and a 6D flying end effector [14] has represented a successful integration of known robotic algorithms, dealing with both motion and interaction control, in a platform with a minimal sensor suite (pose 


\begin{tabular}{|l|l|l|l|l|}
\hline $\begin{array}{l}\text { Aerial } \\
\text { Manipulator }\end{array}$ & $\begin{array}{l}\text { Size } \\
{[\mathrm{m}]}\end{array}$ & $\begin{array}{l}\text { Max. } \\
\text { Total } \\
\text { Mass } \\
{[\mathrm{kg}]}\end{array}$ & $\begin{array}{l}\text { Max. } \\
\text { Flight } \\
\text { Time } \\
{[\mathrm{min}]}\end{array}$ & $\begin{array}{l}\text { Arm(s) configuration } \\
\text { (maximum reach }[\mathrm{m}])\end{array}$ \\
\hline \hline TiltHex & 1.05 & 1.8 & 8 & Fixed bar arm $(0.4 \mathrm{~m})$ \\
\hline AEROX & 2.0 & 25 & 15 & One 6-DoF arm (1 m) \\
\hline $\begin{array}{l}\text { Dual-arm, } \\
\text { stiff joints }\end{array}$ & 1.7 & 18 & 20 & $\begin{array}{l}\text { Dual arm, each with } \\
\text { 5-DoF, stiff joints (0.6 } \\
\text { m) }\end{array}$ \\
\hline $\begin{array}{l}\text { Dual-arm, } \\
\text { compliant } \\
\text { joints }\end{array}$ & 1.2 & 7 & 12 & $\begin{array}{l}\text { Dual arm, each with } \\
\text { 4-DoF, compliant } \\
\text { joints }(0,5 \mathrm{~m})\end{array}$ \\
\hline
\end{tabular}

TABLE I

CHARACTERISTICS OF PLATFORMS DEVELOPED IN AEROARMS

sensor plus IMU) not even needing a force sensor. Stable interaction was obtained also in previous approaches, e.g. in [3] where an aerial manipulator interacts with a vertical surface, but in AEROARMS there are experiments involving complex interaction tasks such as peg-in-hole with tilted holes and sliding on tilted surfaces.

Two main motion control approaches have been considered. The first one is based on modeling the aerial manipulator as an unique system and designing the control scheme on the complete kinematic and dynamic models, see for instance [17] where an integral backstepping controller has been implemented. The second approach considers the aerial platform and the robotic arm as two separate entities and, thus, two autonomous controllers are designed, one for the aerial vehicle, where the effects of the arm on dynamics are handled as disturbances, and one for the arm, where, vice versa, the disturbance is due to the vehicle. Such paradigm has been experimentally exploited, e.g., in [18], where the aerial vehicle controller includes an estimator of external forces and moments to compensate neglected dynamics and the arm dynamics.

Vision-based techniques have also been exploited. In [19] the feedback output from a camera attached to the end effector is adopted in a hierarchical control law. The camera images are exploited to drive the arm end-effector to a desired position and orientation.

One of the AEROARMS objectives is the inspection through contact while flying. This requires to exert forces on a surface in order to maintain the sensor (i.e. ultrasonic) installed in the manipulator end-effector in contact with the surface. Since interaction forces and moments can cause large deviation in motion control and/or destabilize the system, some active and/or passive compliance should be added to the vehicle and the manipulator end-effector. Passive compliance has been adopted in the platform in Fig. 1(d), through the elastic actuators of the compliant arms. This allows to cope efficiently with interaction forces, providing also measurements of these forces that are used to close the feedback loop [16]. Adoption of active compliance approaches requires measurements of the interaction wrenches, that can be achieved, e.g., by using a wrist mounted force/torque sensor on the arm, as in [20], where the contact wrenches are fed to an admittance filter. Such a control scheme has been tested on an underactuated quadrotor equipped with a 6-DoFs manipulator.

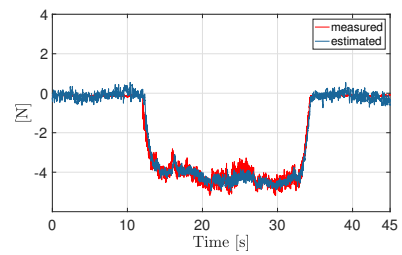

(a) Contact force acting on the end effector

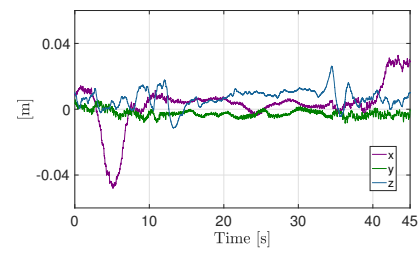

(c) Position tracking error

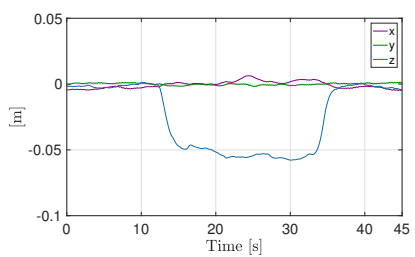

(b) Admittance error

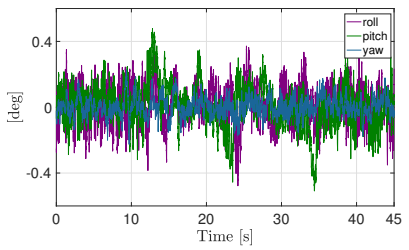

(d) Orientation tracking error
Fig. 3. Experimental results for interaction control

An alternative solution to the force/torque sensor is the use of wrench estimators as proposed in [21] and in [14]. Adoption of under-actuated platforms implies that the lateral forces in body frame, which cannot be provided by the aerial platform itself, have to be generated through the dynamical/inertial coupling between the arm and the aerial robot, which is usually very hard to be handled in an accurate way. To overcome this drawback the admittance control paradigm has been exploited by considering the platform in Fig. 1(a), characterized by noncollinear fixedly-tilted propellers which make possible to control independently the translational and angular acceleration when unconstrained, or any of the six components of the exerted wrench when in contact [14]. As case study has been considered the contact inspection of a pipe and a peg-in-hole emulating a sensor installation. Figure 3 shows the relevant variables for one of the experiments. Videos can be found at http://homepages.laas.fr/afranchi/robotics/?q=node/414 .

Regarding the vehicle-arms coordination, a behavioral control scheme has been proposed for handling both single and dual arm aerial manipulators. The approach in [22], is based on an extension of the set-based null-space-based approach [23] to the case of aerial manipulators, which involves very different challenging issues with respect to other robotic systems. From one hand, the results show that this generalization is successful, on the other hand, specific behaviors and design procedures have been tailored due to the different actuating characteristics.

\section{TELEOPERATION}

In order to evaluate the feasibility of aerial telemanipulation for industrial I\&M tasks, the AEROARMS teleoperation system should ensure: 1) a stable and transparent teleoperation system under the channel characteristics of the wireless communication link, 2) stability of the coupled controller for the manipulator and aerial base. 
Stable teleoperation with communication time delay

For the time-delayed teleoperation system, the trade-offs between stability and performance requirements are tackled by means of a novel four-channel architecture in which force and position signals are exchanged between both the haptic interface (master) and the aerial robot (slave). In each of the channels, a passivity-based controller is exploited to guarantee stability under nominal communication time delays, packet loss or jitter, while transparency is maintained. To this end, a widely used passivity tool, namely, time domain passivity approach (TDPA), has been utilized. The overview of the implemented bilateral controller for the AEROARMS project is shown in Fig. 4.

$\leftrightarrow \begin{gathered}\text { Master } \\ \text { Ctrl. with } \\ \text { Passivity } \\ \text { check }\end{gathered} \leftrightarrow \begin{aligned} & \text { Wireless } \\ & \text { Comm. } \\ & \text { with delay }\end{aligned} \leftrightarrow \begin{gathered}\text { Slave } \\ \text { Ctrl. with } \\ \text { Passivity } \\ \text { check }\end{gathered} \leftrightarrow$

Fig. 4. Teleoperation architecture for the aerial scenario.

Stable coupled control of the manipulator-aerial base system

Stability of the coupled controller for an helicopter with a manipulator is proven in [24] for the autonomous scenario. Here, the manipulator wrench forces are computed in the fuselage frame of the helicopter whereby passivity is ensured. As an extension to the teleoperation case, the passivity check with TDPA to remove the destabilizing effects of communication time delay is applied for the coupled controller. The onground Hardware-In-the-Loop (HIL) simulator [25] is adapted to reproduce the dynamics and control of the aerial system to repeatedly test the bilateral control under defined conditions.

Fig. 5 shows the results of the teleoperation peg-in-hole experiments performed on the HIL with the helicopter control and the dynamic simulation. Although the designed controller produced a stable and high performing system (in terms of pure teleoperation), as it can be seen, the base of the manipulator moves as a result of the manipulator motion and external interaction. It was learned that for highly intricate tasks like precise end-effector positioning and accurate force exertion on the environment, reactive dynamics of the aerial base makes the task completion highly challenging to the operator (note especially the time between $27-30$ seconds in the plots). In order to aid the operator, benefits of task dependent autonomy can be made use of. So, using virtual fixtures [26] and visionbased shared control [27], rather than pure teleoperation are planned during the scope of AEROARMS.

\section{PERCEPTION}

Existing techniques for perception in aerial manipulation are, in most cases, methods adapted from aerial vehicles without manipulation skills to provide positional accuracy and place recognition [28]. The AEROARMS I\&M usecases require however, perception modules that go beyond the state of the art to: (1) accurately localize the vehicle, both during the navigation and manipulation phases; (2) localize
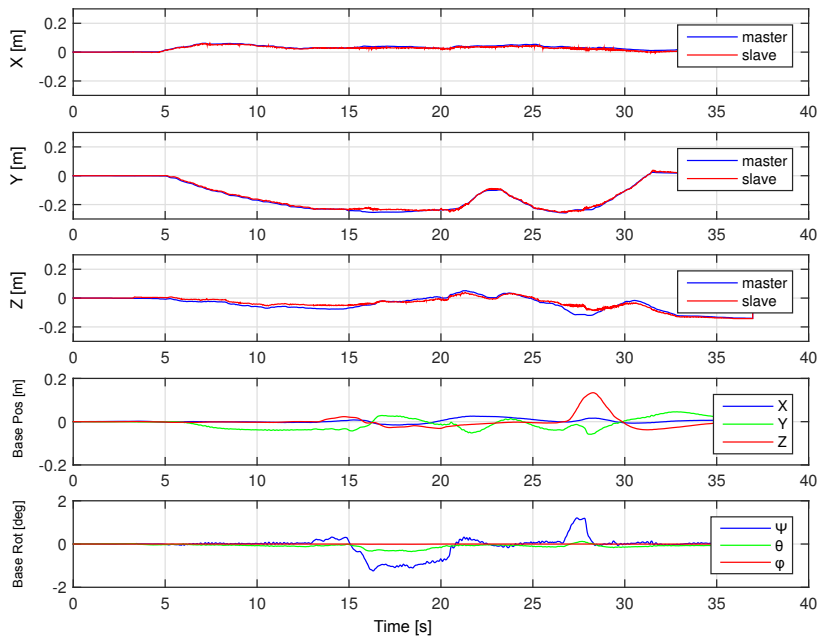

Fig. 5. Position of the master and slave end-effectors (top 3) for a peg-in-hole task experiment with $40 \mathrm{~ms}$ round-trip delay. Notice that in the last 2 plots, the base positions and orientations change significantly with respect to the slave motion

and register target pipes; (3) detect the objects involved in the I\&M tasks and; (4) pick up or release the crawler by the aerial robot. Aerial manipulators are expected to work in realistic industrial scenarios, which are complex, dynamic, unstructured, obstacle-dense and with poor or no GNSS reception, and the techniques to be employed must adapt to unknown dynamic environmental and illumination conditions. Perception techniques must benefit from various sensors with different physical principles and fuse their readings. The ones used in AEROARMS include 3D LIDAR, stereo cameras, IMUs, optical flow sensors and ultra wide band range sensor nodes deployed in the scenario (see Figure 6(a)).

In the AEROARMS I\&M use cases, two operational stages can be distinguished: navigation in the environment and manipulation in close distance to obstacles. Real time accurate and robust 6 DoF pose estimates of the robot are computed on-board by a multi-sensor filter based on Monte Carlo localization (MCL) that integrates the measurements from the 3D LIDAR, the stereo camera and the UWB nodes using a precomputed multi-sensor map.

In flights prior to I\&M missions, a multi-sensor mapping scheme builds separate maps for each sensor and treats them as different layers of the multi-sensor map, all layers sharing the same temporal and spatial frames. We preferred this scheme versus a monolithic approach because it provides higher modularity, flexibility, efficiency and gives capacity to guide the development leveraging the contribution of each sensor.

Figure 6(c) shows the multi-sensor map obtained in the experiment in Fig. 6(b), which was also the testing scenario in Fig. 10. The robot localization computed by the multi-sensor MCL in that experiment had a mean error w.r.t. RTK GPS of $9.9 \mathrm{~cm}$, which was $47 \%$ lower than the robot localization estimation obtained using LOAM [29].

Robot pose estimation during manipulation requires higher levels of accuracy. To that end, the PL-SLAM algorithm 


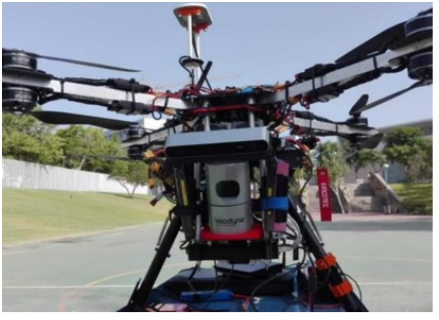

(a)

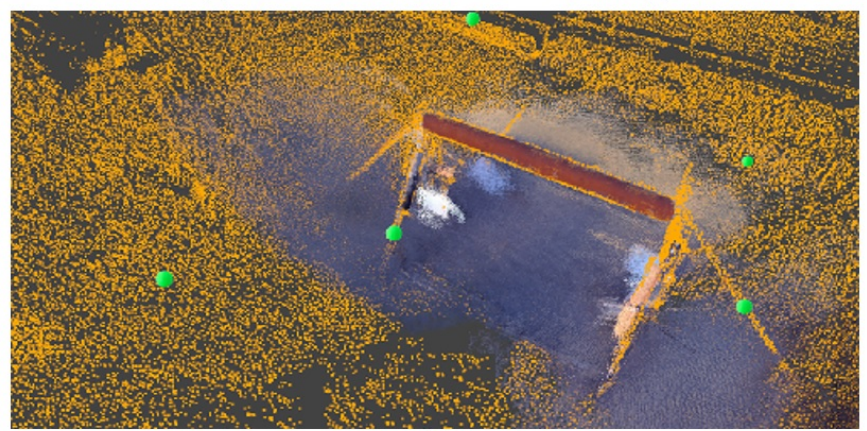

(c)

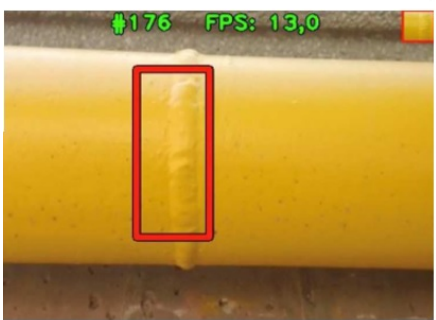

(d)

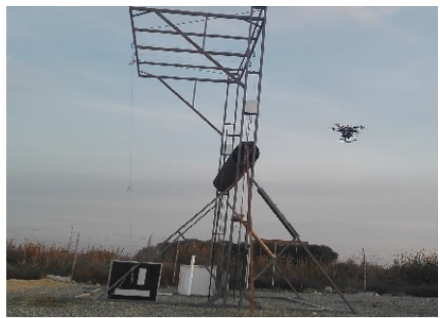

(b)

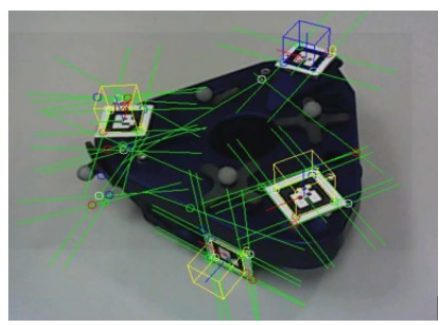

(e)
Fig. 6. Aerial vehicle with multi-sensor setup (a). Picture during mapping experiments (b). Multi-sensor map (c) built with LIDAR -orange dots in the figure, UWB -green dots- and stereo vision. Pipe weld tracking (d). Precise localization of a robot crawler using artificial markers (e).

was developed to leverage the state of the art in SLAM, ORB-SLAM [30], with the simultaneous estimation of point and line features [31], with precise localization at a rate of $3 \mathrm{~Hz}$, resulting in better accuracy than direct methods. Parameterizing lines by their endpoints provides robustness against occlusions and in poorly textured environments. To improve the localization precision a new method using camera images and Deep Learning was used, reaching a precision of $4,5 \mathrm{~cm}$. Fig. 7(a) shows the deep learning architecture. Faster odometry estimates are obtained by fusing IMU and optical flow data. In AEROARMS, sensor synchronization is addressed with time stamps enabling timing errors of some milliseconds, sufficient to obtain the required GNSS-free pose estimation errors.

Once the pipe is localized, the industrial use-cases require to identify and track specific I\&M characteristics and artifacts on the pipe, such as welding marks or corrosion points. The specific characteristics to be tracked can be chosen on-line by an operator, thus off-line learning methods are not feasible due to the low size of the training data set. We developed an on-line semi-supervised boosting method [32] that adapts to real-time changes in illumination, cast shadows or partial occlusions. In this case, the operator selects on-line the type of defect, and the perception system adapts the pattern shape,

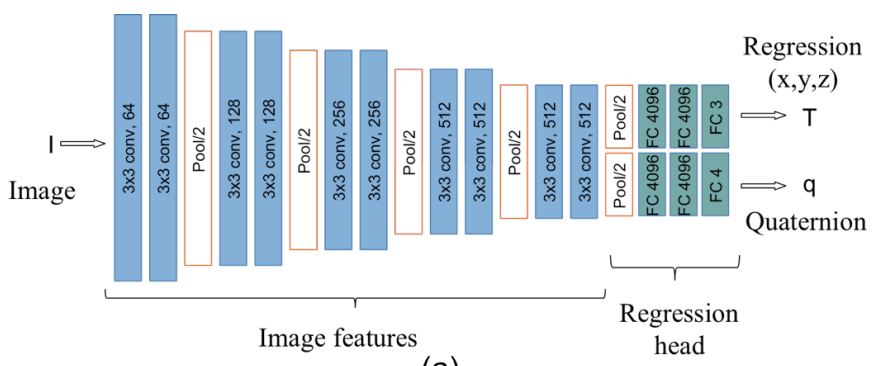

(a)

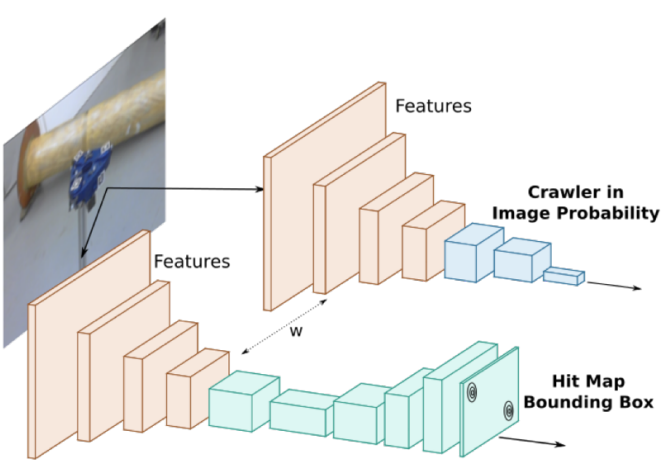

(b)

Fig. 7. Deep learning for precise aerial vehicle localization (a). Deep learning architecture for aerial vehicle localization (b). Deep learning architecture for crawler localization.

color and texture to detect and track the defect. This new method can work in two modes: in the first mode the system is as "on-line learning" and if the defect characteristics change significantly, the system asks the operator to incorporate these new characteristics in the model; and in the second mode, the system works only identifying or tracking the defect, allowing some adaptation, but big changes are not allowed. An example of weld detection and tracking using this method is shown in Fig. 6(d).

The deployment of a robot crawler is also a use case as mentioned above. Pick-up and release of the crawler by the aerial robot is performed in two steps. In the approach maneuver the crawler pose is roughly estimated with an appearance-based Deep Learning method whose architecture is shown in Fig. 7(b) and, for the actual pick up or release, a positioning system based on deformable markers was built [33], guaranteeing very high accuracy, $2 \mathrm{~cm}$ precision as shown in Fig. 6(e).

\section{Motion Planning AND NAVIGation}

Motion planning is required to: 1) move the aerial manipulator from a take-off position to the area to be inspected/maintained; 2) perform the inspection/maintenance task. The former may require navigation in environments cluttered with pipes, structures, etc. (see Fig. 2). The latter requires treating dynamic constraints in a subspace defined by the task. Both methods are presented below.

\section{Navigation in cluttered environments}

A motion planning approach for aerial manipulators exploiting the concept of differential flatness has been developed. 


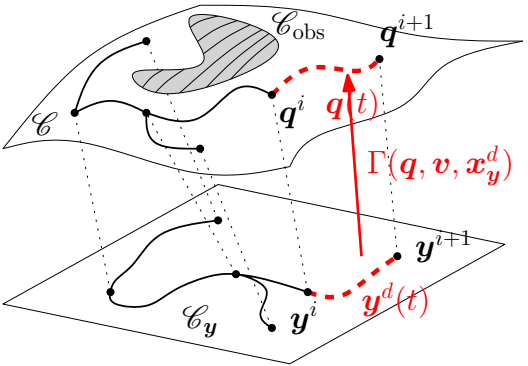

Fig. 8. Schematic representation of the control-aware planning paradigm. To each trajectory in the task space corresponds a motion of the robot is generated by the control method.

Differential flatness is very useful because it allows to plan trajectories directly in a lower dimensional space without the need to consider explicitly the fulfillment of the complex nonlinear and underactuated dynamics of the system. For instance, it has been exploited for dynamic grasping using aerial manipulators with a single-link arm [34]. In the context of AEROARMS, in which an aerial manipulator has to interact by means of the end effector, it results very useful if the end-effector position and orientation belong to a flat output of the system. This is unfortunately not the case for any aerial manipulator. Our main contribution in this field has been to prove that there exists a class of aerial manipulator designs, that we named protocentric, for which this property is ensured [35].

\section{Motion planning for task-constrained aerial manipulation}

An approach that reinforces the connection between motion planning and control in the context of aerial manipulation has been developed [36]. The underlying idea consists in using the controller as a local method to connect neighboring states within a (global) motion planning algorithm. The use of the controller inside the planner guarantees the feasibility of the trajectory for the real system, satisfying task constraints in addition to geometric, kinematic and dynamic constraints. Furthermore, it also allows to better predict the behavior of the closed loop system. Another advantage is that, using a control method that directly treats the redundancy of the system, allows the planner to search for a solution directly in the reduced and more relevant task space. Planning directly at the task level enables a more straight formulation of task-constrained motion planning problems, and generally reduces the dimensionality of the search space. Finally, properly defining the task, one can choose a good trade-off between the dimension of the search space and the delegation of some DoF to the controller.

The proposed control-aware motion planner applies a sampling-based algorithm to search for a solution directly in the task state space $\mathcal{X}_{\boldsymbol{y}}=\mathcal{C}_{\boldsymbol{y}} \times \mathcal{V}_{\boldsymbol{y}} \times \mathcal{A}_{\boldsymbol{y}}$, which in general represents all possible poses $\left(\mathcal{C}_{\boldsymbol{y}}\right)$, velocities $\left(\mathcal{V}_{\boldsymbol{y}}\right)$ and accelerations $\left(\mathcal{A}_{y}\right)$ of the end-effector while constrained to a given task. Figure 8 shows a scheme of the approach.

The planner is able to find trajectories in constrained environments where a purely reactive gradient-based approach fails due to local minima traps. Figure 9 shows one of the scenarios used for the evaluation in simulation reproducing a plausible I\&M application by contact which requires the endeffector to be always in contact and perpendicular to the pipe. The planner requires about 20 seconds to obtain a solution trajectory in this context.

\section{AEROARMS AERIAL ROBOT FOR INDUSTRIAL CONTACT INSPECTION}

Various control, perception and planning algorithms and functionalities have been described in the previous sections. After a research period where different approaches and functionalities were developed and tested in controlled environment, all this know-how has been applied to develop an aerial robot for industrial outdoor contact inspection (see Fig. 10), which is the first use case of AEROARMS.

The main characteristics of this aerial platform, called AEROX, are shown in Table I. It has a 8 rotors configuration with a small angular gap at the front and back of the robot to allow the robotic arm pass between the propellers. Their motors are tilted alternatively 30 degrees around their arms, allowing the control of the lateral accelerations to avoid the tilting of the platform. Moreover, this aerial robot has a special design integrating a new type of aerial robotic manipulator, able to rotate around the vehicle CoM, being capable of robust contact operations that can be used for precise industrial inspections. This novel robotic manipulator provides the following functionalities: orientate the end effector in one degree of freedom (up and down) with 180 working space, reject efficiently the external perturbations (wind, force and torques generated from the contact, etc.), maintain a specific force in contact with the surface and calculate the position of the end effector with respect to the aerial robot.

In order to validate this configuration, many experiments have been conducted. The video at https://youtu.be/vc6E_ $3 i$ S690 shows a summary of the experiments including laboratory tests in an indoor testbed with a motion tracking localization system used as ground truth, outdoor experiments with a safety tether and outdoor free flight experiments.

Fig. 11 shows how multiple perturbations were injected using a rope in order to test its rejection and stability. Each perturbation is easily identified looking to the first graph (attitude) due to the rolling produced each time the rope is

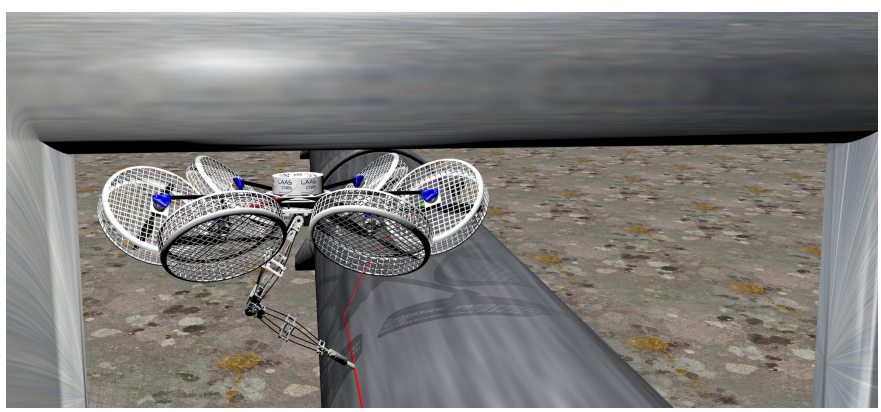

Fig. 9. Illustration of a planned trajectory for pipe inspection. The planned trajectory allows the robot to maintain the contact between the sensor (installed at the end of the arm) and the inspected pipe while avoiding collisions with the other pipe on top. The path followed by the sensor is represented by the red line. 


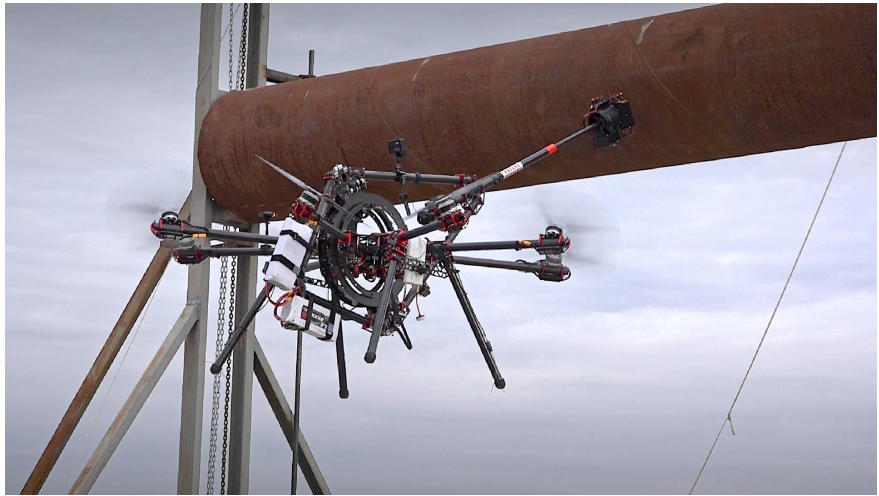

Fig. 10. Outdoor contact inspection experiments with AEROARMS aerial robot

pulled, or even looking to the forces response. The relative position of the robot is validated using the ground truth localization system. As a result, we obtain precise estimations with a RMS error of $9.2 \mathrm{~mm}$.

A teleoperation device, specifically designed for contact inspection operations, has been developed. It is composed of the following elements:

1) Video camera allowing remote view of the interested area;

2) Wheeled system to move the end effector when in contact with the pipe;

3) Inspection sensor: ultrasound or eddy current sensor to perform industrial contact inspections;

4) Ground Interface with camera and sensor device allowing the operator to monitor and modify all the inspection parameters.

Then, the procedure to perform inspections with this novel aerial robot is:

1) The aerial robot takes-off and gets closer to the area to be inspected. This phase could be autonomous or teleoperated and may eventually benefit from the above mentioned trajectory planning;

2) The aerial robot reaches contact with the pipe to be inspected. This phase can also be autonomous or teleoperated and benefits from the perception system;

3) The aerial robot is changed into inspection mode, where the aerial robot automatically maintains its position with respect to the point of contact. From this moment, the inspection operator has full control over the system:

a) The operator, looking at the camera, can move the sensor along the pipe disregarding the individual DoFs of the aerial robot, benefit from the task-priority controller, and eventually resorting to shared control methods;

b) Once at the area of interest, the operator uses the sensor control interface to perform the inspection.

4) The operator can then move the end-effector to the next area of interest, or finalize the inspection;

5) After the complete inspection is finalized, the pilot switches back to flying-mode and de-attaches the aerial robot from the pipe.
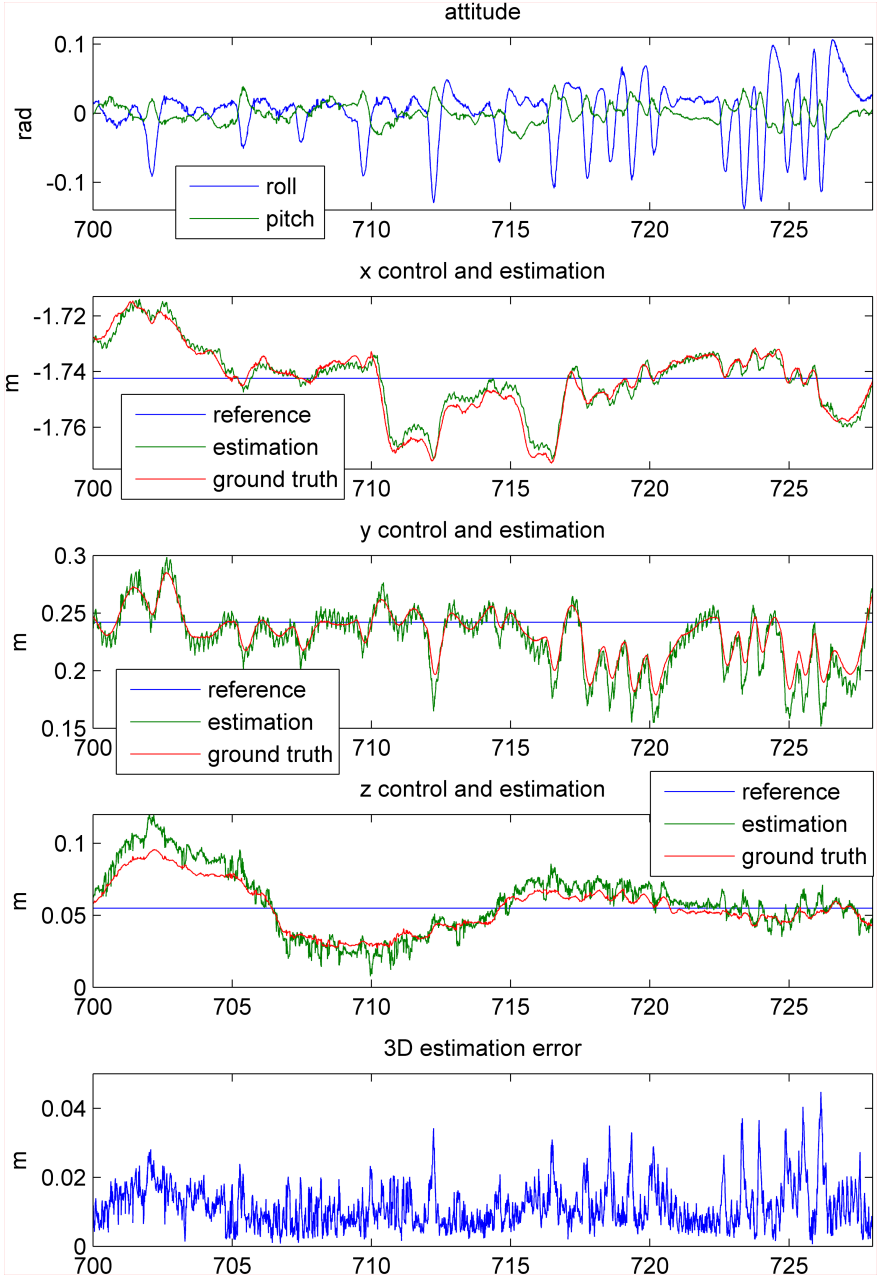

Fig. 11. Indoor autonomous contact operation telemetry during an experiment where 17 strong perturbations up to $100 \mathrm{~N}$ were injected. The attitude graph (top) shows the low effect of the perturbations on the robot attitude. The robot 3D position estimated error in the experiment (bottom) was in average lower than $1 \mathrm{~cm}$ even with these perturbations.

\section{LESSONS LEARNED}

Experiments in industrial settings can only be performed after testing extensively the robustness of the proposed solutions. In this testing, the main lessons learned were:

- Wind and aerodynamic perturbations generated by close surfaces should be compensated by the controller fast enough to maintain safety and achieve accuracy and relative stability. Multi-directional thrust platforms are better suited to maintain forces in any direction as required for contact inspection. Compliance in the arms is also very relevant to maintain safety. Currently available light servo-controllers impose limitations in the accuracy that can be obtained in the end-effector;

- The above mentioned perturbations have significant impact in navigation and manipulation, generating deviations from trajectories planned with conventional methods. The use of a controller inside the planner is useful to predict the dynamic behavior of the closed loop system. Moreover, reactivity methods are needed for dynamic environments or incremental map building; 
- For time-delayed teleoperation, task-dependent shared control algorithms might be helpful to: a) track the master position accurately by the slave manipulator in spite of the base movement, and b) mask the dynamics of the aerial base to the operator so that he perceives a fixed base teleoperation;

- Sensor data fusion methods should be applied to compensate the limitation of individual sensors, such as the loss of GPS, lighting changes and electromagnetic perturbations.

\section{CONCLUSIONS}

AEROARMS has demonstrated the application of aerial robots to contact inspection of industrial pipes while flying, being able to perform ultrasonic measurements to determine the thickness of the pipes wall. The results presented in this paper demonstrated the suitability of aerial robotic manipulation for these outdoor applications. Significant advances with respect to the state of the art have been necessary from design, perception, teleoperation and motion planning and control.

Most of the proposed methods and technologies have been demonstrated outdoor, which is a relevant departure from other published works on aerial robotic manipulation. The technologies of multi-directional thrusters and compliance in the end-effectors have been used in an industrial prototype for the ultrasonic measurements of pipes wall thickness. This work obtained the Overall Innovation Radar Prize 2017 of the European Commission in the ICT event (see http://www.euronews.com/2017/12/04/new-dronetechnology-wins-innovation-radar-prize-2017).

Implementation in factories of the flying-based contact measurement and the deployment of the crawler are in preparation.

\section{ACKNOWLEDGMENTS}

The AEROARMS Consortium consists of 10 European partners (https://aeroarms-project.eu/) led by the Univ. Seville. The funding of the European Commission under the H2020 contract 644271 is acknowledged.

\section{REFERENCES}

[1] P. E. I. Pounds, D. R. Bersak, and A. M. Dollar, "Grasping from the air: Hovering capture and load stability," in 2011 ICRA, May 2011, pp. 2491-2498.

[2] N. Michael, J. Fink, and V. Kumar, "Cooperative manipulation and transportation with aerial robots," Autonomous Robots, vol. 30, no. 1, pp. 73-86, Jan 2011. [Online]. Available: https://doi.org/10.1007/ s10514-010-9205-0

[3] M. Fumagalli, R. Naldi, A. Macchelli, F. Forte, A. Q. L. Keemink, S. Stramigioli, R. Carloni, and L. Marconi, "Developing an aerial manipulator prototype: Physical interaction with the environment," IEEE Robotics Automation Magazine, vol. 21, no. 3, pp. 41-50, Sept 2014.

[4] C. Korpela, M. Orsag, T. Danko, B. Kobe, C. McNeil, R. Pisch, and P. Oh, "Flight stability in aerial redundant manipulators," in 2012 ICRA, May 2012, pp. 3529-3530.

[5] G. Hunt, F. Mitzalis, T. Alhinai, P. A. Hooper, and M. Kovac, "3d printing with flying robots," in 2014 ICRA, May 2014, pp. 4493-4499.

[6] S. Kim, H. Seo, and H. J. Kim, "Operating an unknown drawer using an aerial manipulator," in 2015 ICRA, May 2015, pp. 5503-5508.

[7] T. Bartelds, A. Capra, S. Hamaza, S. Stramigioli, and M. Fumagalli, "Compliant aerial manipulators: Toward a new generation of aerial robotic workers," IEEE Robotics and Automation Letters, vol. 1, no. 1, pp. 477-483, Jan 2016.
[8] H. N. Nguyen, S. Park, and D. Lee, "Aerial tool operation system using quadrotors as rotating thrust generators," in 2015 IROS, Sept 2015, pp. $1285-1291$

[9] H. Seo, S. Kim, and H. J. Kim, "Aerial grasping of cylindrical object using visual servoing based on stochastic model predictive control," in Robotics and Automation (ICRA), 2017 IEEE International Conference on. IEEE, 2017, p. 63626368

[10] D. R. McArthur, A. B. Chowdhury, and D. J. Cappelleri, "Design of the i-boomcopter uav for environmental interaction," in 2017 IEEE International Conference on Robotics and Automation (ICRA), May 2017, pp. 5209-5214.

[11] H. W. Wopereis, J. J. Hoekstra, T. H. Post, G. A. Folkertsma, S. Stramigioli, and M. Fumagalli, "Application of substantial and sustained force to vertical surfaces using a quadrotor," in 2017 IEEE International Conference on Robotics and Automation (ICRA), May 2017, pp. 27042709.

[12] A. Franchi and A. Mallet, "Adaptive closed-loop speed control of bldc motors with applications to multi-rotor aerial vehicles," in 2017 IEEE International Conference on Robotics and Automation (ICRA), May 2017, pp. 5203-5208.

[13] P. Sanchez-Cuevas, G. Heredia, and A. Ollero, "Characterization of the aerodynamic ground effect and its influence in multirotor control," International Journal of Aerospace Engineering, vol. 2017, 2017.

[14] M. Ryll, G. Muscio, F. Pierri, E. Cataldi, G. Antonelli, F. Caccavale, and A. Franchi, "6D physical interaction with a fully actuated aerial robot," in 2017 ICRA. Singapore: IEEE, 2017.

[15] C. M. Korpela, M. Orsag, and P. Y. Oh, "Towards valve turning using a dual-arm aerial manipulator," 2014 IEEE/RSJ International Conference on Intelligent Robots and Systems, pp. 3411-3416, 2014.

[16] A. Suarez, G. Heredia, and A. Ollero, "Physical-virtual impedance control in ultralightweight and compliant dual-arm aerial manipulators," IEEE Robotics and Automation Letters, vol. 3, no. 3, pp. 2553-2560, 2018.

[17] G. Heredia, A. E. Jimenez-Cano, I. Sanchez, D. Llorente, V. Vega, J. Braga, J. A. Acosta, and A. Ollero, "Control of a multirotor outdoor aerial manipulator," in 2014 ICRA, Sept 2014, pp. 3417-3422.

[18] F. Ruggiero, M. Trujillo, R. Cano, H. Ascorbe, A. Viguria, C. Perez, V. Lippiello, A. Ollero, and B. Siciliano, "A multilayer control for multirotor uavs equipped with a servo robot arm," in 2015 ICRA, 2015, pp. 4014-4020.

[19] A. Santamaria-Navarro, P. Grosch, V. Lippiello, J. Solà, and J. AndradeCetto, "Uncalibrated visual servo for unmanned aerial manipulation," IEEE/ASME Transactions on Mechatronics, vol. 22, no. 4, pp. 16101621, Aug 2017.

[20] E. Cataldi, G. Muscio, M. Trujillo, Y. Rodriguez, F. Pierri, G. Antonelli, F. Caccavale, A. Viguria, S. Chiaverini, and A. Ollero, "Impedance control of an aerial-manipulator: Preliminary results," in 2016 IROS. Daejeon, South Korea: IEEE, 2016.

[21] T. Tomic, C. Ott, and S. Haddadin, "External wrench estimation, collision detection, and reflex reaction for flying robots," IEEE Transactions on Robotics, vol. PP, no. 99, pp. 1-17, 2017.

[22] G. Muscio, F. Pierri, M. Trujillo, E. Cataldi, G. Antonelli, F. Caccavale, A. Viguria, S. Chiaverini, and A. Ollero, "Coordinated control of aerial robotic manipulators: theory and experiments," IEEE Transactions on Control Systems Technology, 2017.

[23] S. Moe, G. Antonelli, A. Teel, K. Pettersen, and J. Schrimpf, "Set-based tasks within the singularity-robust multiple task-priority inverse kinematics framework: General formulation, stability analysis and experimental results," Frontiers in Robotics and AI, vol. 3, p. 16, 2016.

[24] M. Kim, R. Balachandran, M. De Stefano, K. Kondak, and C. Ott, "Passive compliance control of aerial manipulators," in Submitted to Intelligent Robots and Systems (IROS), 2018 IEEE/RSJ International Conference on. IEEE.

[25] J. Artigas, M. D. Stefano, W. Rackl, R. Lampariello, B. Brunner, W. Bertleff, R. Burger, O. Porges, A. Giordano, C. Borst, and A. AlbuSchaeffer, "The oos-sim: An on-ground simulation facility for on-orbit servicing robotic operations," in 2015 ICRA, May 2015, pp. 2854-2860.

[26] J. J. Abbott, P. Marayong, and A. M. Okamura, "Haptic virtual fixtures for robot-assisted manipulation," in Robotics research. Springer, 2007, pp. 49-64.

[27] F. Abi-Farraj, N. Pedemonte, and P. R. Giordano, "A visual-based shared control architecture for remote telemanipulation," in Intelligent Robots and Systems (IROS), 2016 IEEE/RSJ International Conference on. IEEE, 2016, pp. 4266-4273.

[28] M. Karrer and M. Chli, "Towards globally consistent visual-inertial SLAM for collaborative aerial navigation," in Proc. of the IEEE Int. Conf. on Robotics \& Automation (ICRA), 2018. 
[29] J. Zhang and S. Singh, "LOAM: Lidar odometry and mapping in realtime," in Robotics: Science and Systems Conference, Pittsburgh, PA, July 2014.

[30] R. Mur-Artal, J. Montiel, and J. Tardós, "ORB-SLAM: A versatile and accurate monocular SLAM system," IEEE Trans. on Robotics, vol. 31, no. 5, pp. 1147-1163, Oct 2015 .

[31] A. Pumarola, A. Vakhitov, A. Agudo, A. Sanfeliu, and F. MorenoNoguer, "PL-SLAM: Real-time monocular visual slam with points and lines," in Proc. IEEE Int. Conf. Robotics and Automation, May 2017, pp. $4503-4508$.

[32] M. Villamizar, J. Andrade-Cetto, F. Moreno-Noguer, and A. Sanfeliu, "Boosted random ferns for object detection," IEEE Trans. Pattern Anal. Mach. Intell., 2017.

[33] A. Amor, A. Ruiz, F. Moreno-Noguer, and A. Sanfeliu, "On-board real-time pose estimation for uavs using deformable visual contour registration," in 2014 IEEE Int. Conf. on Robotics and Automation, Hong Kong, May 2014, pp. 2595-2601.

[34] J. Thomas, G. Loianno, J. Polin, K. Sreenath, and V. Kumar, "Toward autonomous avian-inspired grasping for micro aerial vehicles," Bioinspiration \& Biomimetics, vol. 9, no. 2, p. 025010, 2014.

[35] B. Yüksel, G. Buondonno, and A. Franchi, "Differential flatness and control of protocentric aerial manipulators with any number of arms and mixed rigid-/elastic-joints," in 2016 IEEE/RSJ Int. Conf. on Intelligent Robots and Systems, Daejeon, South Korea, Oct. 2016, pp. 561-566.

[36] M. Tognon, E. Cataldi, H. A. T. Chavez, G. Antonelli, J. Cortés, and A. Franchi, "Control-aware motion planning for task-constrained aerial manipulation," IEEE Robotics and Automation Letters, vol. 3, no. 3, pp. $2478-2484,2018$. 